\title{
Magnetic Fields in the Solar Nebula and the Angular Momentum Transfer
}

\author{
M. Kiguchi ${ }^{1)}$, S. Narita ${ }^{2)}$, T. Terasawa ${ }^{3)}$ and C. Hayashi ${ }^{4)}$ \\ 1) Research Institute for Science and Technology, Kinki University, Osaka, 577, Japan \\ 2) Department of Electronics, Doshisha University, Kyoto, 602, Japan \\ 3) Department of Geophysics, Kyoto University, Kyoto, 606, Japan \\ 4) Kyoto University, Kyoto, 606, Japan
}

\section{Introduction}

We are now carrying out the project to investigate how the primodial solar disk is formed and evolves. One of the central problems of this project is to study the angular momentum transfer in the nebula caused by the magnetic braking or the turbulent mixing in the boundary layer between the surface of the disk and its high temperature coron a envelope.

Here, we summarize the result of analysis for the magnetic braking in 1-dimensional approximation, which will make the basis to interpret the full numerical calculations which we are now carrying out.

\section{Angular Momentum Transport in the Radial Direction}

As pointed out by Hayashi (1981), when seed magnetic fields which are generated by turbulences have radial component, azimuthal component grows by rotation and decays by Joule loss. For this process, the basic equation is given by

$$
\left\{\frac{\partial}{\partial t}+\Omega_{0}\left[r_{0}-\frac{3}{2}\left(r-r_{0}\right)\right] \frac{\partial}{r_{0} \partial \phi}-\frac{c^{2}}{4 \pi \sigma_{e}} \nabla\right\}\left(\begin{array}{c}
H_{r} \\
H_{\phi} \\
H_{z}
\end{array}\right)=\left(\begin{array}{c}
0 \\
-\frac{3}{2} \Omega_{0} H_{r} \\
0
\end{array}\right) .
$$

The azimuthal component $H_{\phi}$ takes the maximum value at $t \sim t_{\text {decay }}, H_{\phi}^{\max } \simeq\left(t_{\text {decay }} / t_{\text {Kepler }}\right) H_{r}(0)$, where $t_{\text {decay }}$ is the decay time $t_{\text {decay }}=4 \pi \sigma_{e} z_{0}^{2} / c^{2}, z_{0}$ is the scale height of the disk, and $t_{\text {Kepler }}$ is the Kepler time. The amplification factor $t_{\text {decay }} / t_{\text {Kepler }}$ takes the value about $10^{-1}-10^{1}$ at Jupiter. The angular momentum is transferred by the $r-\phi$ component of the Maxwell stress tensor along the r-direction. The dynamical viscosity coefficient in this case is given by $\nu \sim\left(t_{\text {decay }} / t_{\text {Kepler }}\right) c_{s} z_{0}$.

\section{Angular Momentum Transport by Alfvén Wave}

Recently, Terasawa et al. (unpublished) has pointed out the following mechanism: When the magnetic fields are uniform in the $z$-direction, the $\phi$-component of magnetic fields which are generated by rotation is transmitted as the Alfven wave in the $z$-direction and the the angular momentum escapes along the $z$-direction. 
In 1-dimensional approximation, it is assumed that $\partial / \partial r=0, v_{z}=0, H_{z}=$ const. , where $v_{z}$ is the z-component of the fluid velocity. The basic equation in this approximation is given by

$$
\frac{\partial h}{\partial t}=\frac{\partial}{\partial t}\left(v_{\phi}+D \frac{\partial h}{\partial z}\right) \quad \frac{\partial v_{\phi}}{\partial t}=v_{\AA}^{2} \frac{\partial h}{\partial z}
$$

where $h=H_{\phi} / H_{z}$ is the $\phi$-component of magnetic fields normalized by the uniform fields, $D=$ $c^{2} / 4 \pi \sigma_{e}$ is the magnetic diffusion coefficient, $\sigma_{e}$ is the electric conductivity, and $v_{\mathrm{A}}=\sqrt{H_{z}^{2} / 4 \pi \rho}$ is the Alfvén velocity. The boundary conditions are given as, at $z=0, h=0, \partial v_{\phi} / \partial z=0$, and at $z \rightarrow \infty$, where the Alfvén velocity is constant and the diffusion coefficient vanishes, $v_{\phi} / v_{\mathrm{A}}=-h$ (out-going wave condition).

The Alfvén velocity $v_{\mathrm{A}}$ and the diffusion coefficient $D$ are different in the disk and in the envelope. In the case of thin transition layer, i.e., $D=D_{0}, v_{\mathrm{A}}=v_{\mathrm{A} \text {,disk }}$ for $z<z_{0}$, and $D=0, v_{\mathrm{A}}=v_{\mathrm{A} \text {,ext }}$ for $z>z_{0}$, we can easily find a solution implicitly which has the functional form (compare with the solution by Mouschovias and Paleologou) of $h=-h_{0} \mathrm{e}^{-\lambda t} \sinh (k z), v_{\phi}=-v_{0} \mathrm{e}^{-\lambda t} \cosh (k z)$ in the disk. From the differential equation, we get $\lambda / k^{2}=v_{\AA \text {,disk }}^{2} / \lambda-D_{0}$ and $h_{0} / v_{0}=k / \lambda$. From the boundary condition, we get $\tanh \left(k z_{0}\right)=v_{0} / h_{0} v_{\mathrm{A} \text {,ext }}=\lambda / k v_{\mathrm{A} \text {,disk }}$. If the density contrast between disk and envelope is very large, so that $v_{\mathrm{A}, \mathrm{disk}} / v_{\mathrm{A} \text {,ext }}<<1$, the solution becomes

$$
\lambda=\frac{v_{\mathrm{A}, \mathrm{disk}}^{2}}{z_{0} v_{\mathrm{A}, \mathrm{ext}}+D}, \quad k^{2}=\frac{v_{\mathrm{A}, \mathrm{disk}}^{2}}{z_{0} v_{\mathrm{A}, \mathrm{ext}}\left(z_{0} v_{\mathrm{A}, \mathrm{ext}}+D\right)}, \quad \frac{v_{\phi}\left(z=z_{0}+\epsilon\right)}{v_{\phi}(z=z-\epsilon)}=1-\frac{\lambda D}{v_{\mathrm{A}, \mathrm{disk}}^{2}}=\frac{z_{0} V_{\mathrm{A}, \mathrm{ext}}}{z_{0} V_{\mathrm{A}, \mathrm{ext}}+D} .
$$

The solution for $z>z_{0}$ is given by $\left.h=h_{0} k z_{0} \mathrm{e}^{-\lambda\left(t-z / v_{\Lambda}, \text { ext }-z_{0} / v_{\Lambda}, \text { ext }\right.}\right) \theta\left(-z+v_{\mathrm{A}} t+z_{f}\right), v_{\phi}=$ $v_{0} \mathrm{e}^{-\lambda\left(t-z / v_{\mathrm{A}, e x t}-z_{0} / v_{\mathrm{A}, \mathrm{ext}}\right)} \theta\left(-z+v_{\mathrm{A}} t+z_{f}\right)$, where $\theta$ is the step function and $z_{f}$ is the position of a wave front at $t=0$. From these results, we can express the in-fall velocity of the gas in Kepler disks as

$$
t_{r}=-\frac{r}{v_{r}}=\frac{D+v_{\mathrm{A}, \mathrm{ext}} z_{0}}{2 v_{\mathrm{A}, \mathrm{disk}}} .
$$

Narita et al. (in preparation) has studied the characteristic features of this mechanism in the proto-solar disk. In actual disks, the surface part is ionized by cosmic rays but the inner part is less ionized, so that the electric conductivity $\sigma_{e}$ depends on $z$. The density $\rho$ in a gravitationally equilibrium disk varies exponentially, so that the Alfvén velocity depends on $z$. In this case, it is not clear how we can modify eq.(4), nor it dose not depend on time. Narita et al. studied these problems numerically.

Numerical results show that

$$
\alpha(z)=\frac{1}{1+\frac{D}{z v_{\mathrm{A}, \mathrm{ext}}}}
$$

which describes a velocity gap $v_{\phi, e x t} / v_{\phi, 0}$, varies timely. If the magnetic fields are scarcely frozen $(\alpha<<1)$, the damping time scale for $v_{\phi}(z)$ is given locally by

$$
\tau(z)=\frac{c^{2} \rho(z)}{H_{z}^{2} \sigma_{e}(z)} \text {. }
$$

This means that when $\alpha<<1$, not only the magnetic braking dose not depend on the external density, but also it does not depend global structure of the disk. From this equation, it is expected that the magnetic braking is effective in the transition region between disk and envelope. This means that the in-fall flow of the gas is fast at the surface region of the disk.

\section{References}

Hayashi, C., 1981, Prog. Theor. Phys., suppl. 70, 35.

Mouschovias, T.C., Paleologou, E.V., 1979, Ap. J. 230, 204. 